\title{
Estudio de caso único sobre la participación de estudiantes en el aula desde los principios del liderazgo distribuido a través de focus group y entrevista semiestructurada aplicada a estudiantes y docentes
}

\author{
Unique case study on the participation of students in the classroom from the principles \\ of distributed leadership through focus group and semi-structured interview applied to \\ students and teachers
}

Estudo de caso único sobre a participação de alunos em sala de aula a partir dos princípios da liderança distribuída por meio de grupos focais e entrevistas semiestruturadas aplicadas a alunos e professores

\author{
Cristóbal Andrés Muñoz Pareschi ${ }^{a}$ \\ ${ }^{a}$ Liceo Salesiano Manuel Arriarán Barros, Chile. \\ crmunoz@boscolab.cl
}

\begin{abstract}
RESUMEN
Los cambios estructurales que enfrenta el mundo como consecuencia de un proceso globalizador al que la educación no queda ajena, nos insta a responder a las nuevas exigencias educativas a través de la mejora en los aprendizajes, siendo necesario dejar atrás la perspectiva tradicional de enseñanza que relega la participación de los estudiantes en el aula a determinaciones por parte del profesor. En este contexto, aparece el Liderazgo Distribuido como un estilo de liderazgo transformador que permite la participación, democratización y corresponsabilidad de los resultados escolares. Teniendo a la base los principios del Sistema Preventivo, es fundamental relacionar fundamentos que caracterizan este liderazgo con la participación de los estudiantes en las Comunidades Educativo Pastorales. El presente estudio pretende comprender la relación entre la participación de estudiantes que cursan el nivel de Enseñanza Media en un Colegio Salesiano de la Región Metropolitana de Santiago-Chile y los principios del Liderazgo Distribuido Su metodología se inspira bajo un paradigma cualitativo, con un diseño de estudio de caso único, descriptivo y exploratorio, aplicando entrevistas estructuradas en profundidad, grupo focal y análisis de documento. Entre los principales hallazgos destaca que existe armonía entre la participación de los estudiantes en ámbitos académicos y de convivencia con los principios del Liderazgo Distribuido centrado en el aula, invisibilizándose la perspectiva tradicional de la participación de los estudiantes relegada a decisiones excluyentes por parte de los profesores.
\end{abstract}

Palabras claves: Liderazgo Distribuido, Sistema Preventivo, Participación, Aula.

\begin{abstract}
The structural changes that the world faces as a consequence of a globalizing process to which education is not alien, urges us to respond to the new educational demands through improved learning, being necessary to leave behind the traditional perspective of teaching that relegates the participation of students in the classroom to determinations by the teacher. In this context, Distributed Leadership appears as a transformative leadership style that allows participation, democratization and co-responsibility for school results. Based on the principles of the Preventive System, it is essential to relate the fundamentals that characterize this leadership with the participation of students in the Educational Pastoral Communities. The present study aims to understand the relationship between the participation of students who attend the High School level in a Salesian School of the Metropolitan Region of Santiago-Chile and the principles of Distributed Leadership. Its methodology is
\end{abstract}


inspired by a qualitative paradigm, with a design of unique, descriptive and exploratory case study, applying in-depth structured interviews, focus group and document analysis. Among the main findings, it stands out that there is harmony between the participation of students in academic and coexistence areas with the principles of Distributed Leadership centered in the classroom, making invisible the traditional perspective of student participation relegated to exclusive decisions by teachers.

Key words: Distributed Leadership, Preventive System, Participation, Classroom.

\section{RESUMO}

As mudanças estruturais que o mundo enfrenta em consequência de um processo globalizante ao qual a educação não é alheia, nos impele a responder às novas demandas educacionais por meio da melhoria da aprendizagem, sendo necessário deixar para trás a perspectiva tradicional de ensino que relega. a participação dos alunos em sala de aula às determinações do professor. Nesse contexto, a Liderança Distribuída surge como um estilo de liderança transformador que permite a participação, democratização e corresponsabilidade pelos resultados escolares. Com base nos princípios do Sistema Preventivo, é fundamental relacionar os fundamentos que caracterizam esta liderança com a participação dos alunos nas Comunidades de Pastoral Educacional. O presente estudo tem como objetivo compreender a relação entre a participação dos alunos do Ensino Médio em uma Escola Salesiana da Região Metropolitana de Santiago-Chile e os princípios da Liderança Distribuída, cuja metodologia se inspira em um paradigma qualitativo, com desenho de estudo de caso único, descritivo e exploratório, com aplicação de entrevistas estruturadas em profundidade, focus group e análise documental. Dentre os principais achados, destaca-se que há harmonia entre a participação dos alunos nas áreas acadêmicas e de convivência com os princípios da Liderança Distribuída centrada na sala de aula, tornando invisível a perspectiva tradicional de participação dos alunos relegada às decisões exclusivas dos profesores.

Palavras-chave: Liderança Distribuída- Sistema Preventivo- Participação- Sala de Aula.

\section{INTRODUCCIÓN}

La presente investigación aborda la relación entre la participación de los estudiantes y principios del Liderazgo Distribuido centrado en el aula de Enseñanza Media de un colegio salesiano de la Región Metropolitana. En la primera parte, marco teórico, se expone la interrogante sobre la participación de los estudiantes en un contexto salesiano y su relación con aspectos del liderazgo distribuido. Para dar respuesta desde la teoría, se detallan conceptos claves que permiten sustentar el estudio y acercarse al cumplimiento de los objetivos, es por eso que, se presenta la Globalización, el Liderazgo Distribuido, la Participación de Estudiantes y el Sistema preventivo de Don Bosco. En la segunda parte, se expone la metodología, donde se define y justifica el enfoque de la investigación, para la cual se consideró el paradigma cualitativo, además, se explica el estudio de caso como el diseño de la investigación. A partir de ahí, se define y describe la muestra teórica y el procedimiento de recolección de los datos. La tercera parte está compuesta por la presentación y análisis de los resultados. En el último apartado, se expone la discusión entre la teoría y el análisis de los datos, para luego concluir el artículo, enfatizando en sus aportes para futuras investigaciones, como también sus limitaciones.

\section{MARCO TEÓRICO}

Hoy en día el mundo vive constantes cambios estructurales, los cuales se han generado con una impensada inmediatez, característica esencial del proceso globalizador, considerando 
que este afecta la estructura social, económica, política y cultural de las naciones. En este ambiente, surgen nuevas problemáticas, porque las sociedades se convierten en fenómenos cada día más difíciles de comprender, debido a la heterogeneidad de los grupos sociales que genera esta denominada aldea global. Es lógico que la educación, como elemento clave del engranaje de las sociedades quede sujeta a los cambios, constituyéndose además como elemento principal para la adaptación humana a este nuevo contexto. Debido a la situación descrita, los desafíos de la educación han cambiado, quedando atrás la preocupación solitaria por los aspectos cognitivos, porque se requiere poner el acento y toma de decisiones en una mirada holística del proceso, considerando en ello elementos interrelacionados que constituyen el proceso de enseñanza para el logro de los aprendizajes. La educación como concepto que denota un proceso donde interactúan sujetos con el objetivo de que se produzca aprendizaje es complejo, por lo que los esfuerzos siempre se han situado en definir elementos esenciales que la constituyen. Maturana (2001), afirma que este proceso está sujeto a cambios constantes, dependiendo de la interacción del organismo, lo que dura desde su inicio hasta su muerte, es decir, un proceso histórico, por lo que un organismo nunca está determinado dese su origen.

Tomando en cuenta lo anterior, en el contexto mundial, existe la interrogante central de cómo mejorar los aprendizajes. A partir de esto las organizaciones educativas apuntan a distintos factores para lograr dicho objetivo. Murillo y Martínez (2018), sostienen la importancia de los factores de aula para un desarrollo integral de los estudiantes. Como consecuencia de lo anterior, el liderazgo se posiciona como un elemento central para la dirección de procesos académicos y administrativos cuya eficacia y pertinencia del tipo que se ejerce, dependerá del contexto de cada organización. Bajo esta perspectiva, el liderazgo según Leithwood (2009), es una práctica que incide en el mejoramiento de la organización, porque conmina a las personas a trabajar para lograr objetivos acordados. Como complemento, Robinson, Lloyd y Rowe (2012), estudian el impacto de los diferentes tipos de liderazgo en los resultados de los estudiantes, académicos y no académicos, para ello, analizan 27 estudios publicados sobre la relación entre liderazgo y resultados de los estudiantes. Maureira (2017), presenta la relevancia que posee el liderazgo en sus diversas acepciones, describiendo y organizando una muestra de dimensiones de la práctica de liderazgo y su evolución conceptual. Desde las organizaciones educativas, las tendencias en el conocimiento del liderazgo destacan que la visión exclusivamente individual está llegando a su fin, así surge la importancia del liderazgo distribuido, el cual supone una distribución del conocimiento. A partir de lo anterior, una práctica de liderazgo distribuido posee dos elementos esenciales: las prácticas de liderazgo y en segundo lugar el reconocer que liderar una escuela va más allá del director, porque existen otros líderes que deben ser incluidos y que deben ser designados, considerando que no tienen un cargo de liderazgo formal (Spillane y Ortiz, 2016). En el caso chileno, se han desarrollado diferentes estudios que comprueban la dificultad para establecer este tipo de liderazgo, en una cultura donde se concentra el poder y donde predomina una visión individual y jerárquica del liderazgo (Ahumada, González, Pino y Maureira, 2017). A pesar del obstáculo planteado por los autores, estos últimos años se ha propuesto el ejercicio del liderazgo distribuido desde una mirada democrática de la escuela, en donde se incluya la participación de todos los actores de la comunidad para alcanzar aprendizajes de calidad. Bajo esta lógica, tanto los líderes como sus seguidores realizan prácticas que deben ser comprendidas desde un contexto o situación específica de interacción que tiene cierta influencia en las respuestas 
Estudios Pedagógicos XLVI N $3: 167-180,2020$

ESTUDIO DE CASO ÚNICO SOBRE LA PARTICIPACIÓN DE ESTUDIANTES EN EL AULA DESDE LOS PRINCIPIOS DEL LIDERAZGO DISTRIBUIDO A TRAVÉS DE FOCUS GROUP Y ENTREVISTA SEMIESTRUCTURADA APLICADA...

que dan unos y otros (Leithwood, 2009). Según Cayulef (2007), el liderazgo distribuido transforma la cultura de la escuela, porque permite la participación, democratización y corresponsabilidad de los resultados escolares. En este sentido, este tipo de liderazgo incluye a todos los actores de la organización educativa, cada uno aportando desde sus distintas funciones y habilidades para el logro de objetivos comunes y, para lo cual, son necesarios distintos cambios que logren la mejora de los aprendizajes. Valorando la gran importancia que tiene el tomar en consideración las distintas situaciones e interacciones que ocurren dentro del aula y que no siempre responden al ámbito académico, DarlingHammond (2001), afirma que es fundamental considerar las distintas perspectivas y experiencias, porque esto genera por una parte el poder de pensamiento de los estudiantes y su visión en cuanto a su función dentro de la sociedad. Relacionado a ello, considerar la experiencia de los estudiantes por parte de los profesores conjugada con su capacidad de conectarla con los contenidos académicos mejoraría el rendimiento académico debido a que el estudiante se sentiría parte en la construcción de sus propios aprendizajes, los cuales serían más cercano a sus propias realidades. Seashore (2017) como argumento de lo anterior, esboza una crítica a la estructura tradicional de la escuela afirmando que las reformas educacionales tanto en Estados Unidos como en otros países se basan en estudios que otorgan gran relevancia al trabajo de los docentes, excluyendo otros actores de las organizaciones que se ubican tanto dentro como fuera de ella. Lo expuesto por el autor, responde a la mirada tradicional de que el docente es el responsable de la enseñanza por lo que la variación de los aprendizajes y por ende los resultados académicos recaen directamente en el cuestionamiento de la calidad de su práctica pedagógica. Esta visión está muy arraigada en la sociedad por lo que se dejan fuera de análisis factores que son trascendentales para dicho objetivo. Si bien el rol del docente es primordial dentro del aula, debido a que se constituye como una figura de líder, eso no basta, el liderazgo integrador se basa en la colaboración, derribando fronteras entre docente y estudiante que antiguamente eran impensadas de eliminar. Finalmente, este liderazgo tiene como piedra angular la interacción, el diálogo y por consiguiente la integración de distintos actores para lograr los objetivos. En este ambiente, el rol del estudiante se hace fundamental a partir de su participación directa en la construcción de su propio aprendizaje.

Uno de los elementos claves para que se ejerza liderazgo distribuido en las organizaciones educativas es el establecimiento de relaciones sociales que promuevan múltiples y diversas interacciones. Weinstein (2019), plantea que la confianza está muy vinculada a la posibilidad de mejorar la escuela, porque tiene que ver con la interdependencia de la escuela para la obtención de resultados positivos, por lo que se constituye como un factor clave dentro de las organizaciones educativas para la mejora. En este sentido, estas relaciones basadas en la confianza se desarrollan entre todos los actores de la comunidad, directivos y docentes, entre docentes, estudiantes y docentes, entre docentes y familia, siempre con el objetivo de la mejora. Relacionado a esto, es fundamental, antes de tratar la participación de los estudiantes, conocer la cultura juvenil y de esta forma comprender desde sus formas de pensar la relación que tienen con el proceso educativo. Lechner (2004), afirma que la cultura juvenil es parte fundamental de la convivencia social. En su estudio, aborda los factores que condicionan las experiencias e imágenes que tienen los jóvenes de la sociedad, tomando en cuenta siempre la heterogeneidad de ellos. Siguiendo esta idea, un espacio de socialización básica de los jóvenes es justamente la escuela, a pesar de que sostiene que el papel de ella en ese sentido se ha ido debilitando y se preocupa mucho de la 
transmisión de conocimientos y formación de habilidades, volviéndose muy instrumental a los requerimientos del sistema productivo. La tesis central del autor es que la relación del joven con la escuela ha cambiado debido a que las reformas han avanzado muy poco en producir socialización vertical y lateral, lo que se traduce en la interacción de los profesores con los estudiantes tanto dentro como fuera de las aulas. Como consecuencia de esto, es fuera de la escuela donde los jóvenes adquieres conocimientos adecuados a su realidad, porque consideran que este espacio educativo carece de sentido. Según Bertoglia (2005), las interacciones en el aula no pueden ser vistas como de causa-efecto, en la que sólo el docente es activo en la entrega de contenidos y los estudiantes son pasivos receptores de información. Al contrario, la relación que se debe dar es de interdependencia, en la que uno de los sujetos estimula la acción del otro. Para que se produzca esta situación ideal es necesaria la participación conjunta, lo que genera una influencia simultánea y recíproca entendida como interacción dialógica constante, donde tanto profesores como estudiantes comparten sus puntos de vista sobre una idea común. Hasta el momento, se ha expuesto acerca de la educación como un proceso social que requiere de interacción constante para que se produzca participación de los estudiantes para la construcción de sus aprendizajes dentro del aula, lo que se produce a través del diálogo con el profesor. Fundamentando esta idea, Escobar (2015) sostiene que estas percepciones determinan la forma en que los estudiantes viven el proceso de enseñanza-aprendizaje, porque el punto de vista del estudiante influye en sus percepciones o estilos particulares de aprender y por ende de actuar y relacionarse en la escuela. De esta forma la interacción entre el alumno y el docente es especial, porque si bien se comprende y acepta que es fundamental centrar la atención en el comportamiento del alumno y del docente, igual de necesario e importante es comprender las apreciaciones que estos realizan en el aula y que por lo general manifiestan de forma explícita. Jurado (2009) desde una visión más política de la escuela, afirma que, en los últimos años, la importancia que se le está dando a la participación de los diferentes actores de la comunidad educativa, ya sea, en la gestión como en la participación de la propia vida del centro, está viviendo un gran momento de valoración positiva, porque tanto el aprendizaje como el desarrollo de actitudes participativas implica para el alumnado garantizar la coherencia con el sistema democrático. Lo que hace capaz al estudiante de reivindicar sus demandas e intereses tanto a nivel local de su escuela como a nivel social, intentando conseguir acuerdos y tomando decisiones compartidas y producidas a través del dialogo con los demás actores de la comunidad. En este sentido, la participación en la escuela pasa a ser un ejercicio previo para que los estudiantes practiquen valores como el respeto, la tolerancia, la igualdad y la valoración de la libre expresión. Elmore (2010) aborda la participación desde el concepto del núcleo pedagógico, el cual está construido en base a la relación entre el profesor, el alumno y los contenidos, donde cada uno de ellos tiene su propio rol y sus propios recursos que aportar al proceso educativo. Considerando su premisa fundamental, para el autor sólo hay tres formas desde el rol del profesor de mejorar los aprendizajes escolares. En primer lugar, aumentando el nivel de conocimientos. En segundo lugar, incrementando la complejidad de contenidos que los estudiantes deben aprender. En tercer y último término, transformando el trabajo de los alumnos en el proceso pedagógico (participación). Todo lo que esté fuera del núcleo pedagógico, sólo influye instrumentalmente sobre los tres elementos. En Chile, relacionado a la importancia de considerar los factores que componen el núcleo pedagógico para generar mejoras para el logro de aprendizajes, Aravena, Escare y Ramírez (2019), exponen la relevancia que 
tiene la participación democrática de los estudiantes, porque parten de la base que esta considera a todos los miembros que componen la escuela como sujetos de derecho con responsabilidades específicas de acuerdo a su rol. Prieto (2005) da cuenta de un estudio sobre la participación de los estudiantes y sus representaciones sobre sus procesos formativos en cuatro escuelas chilenas, concluyendo que existen desafíos en la educación actual, porque el mejoramiento de la calidad va de la mano con el hecho de comprender lo que sucede específicamente en las aulas, esto obliga a tomar en consideración la opinión de los estudiantes, lo cual se debe realizar a través del diálogo y la reflexión, transformando el aula desde su tradicional espacio de poder a uno democrático, valorando la capacidad de los estudiantes en sus habilidades autónomas y críticas, que construyen su posición en la sociedad.

En el contexto de un Colegio Salesiano, la participación de los estudiantes armoniza con principios del liderazgo distribuido, porque los PEPS locales reafirman el espacio educativo de la escuela y del aula en base a la religión, el amor y la razón, que se materializa a través del diálogo constante entre todos los actores de las comunidades educativas para construir en conjunto el aprendizaje de los estudiantes. En este sentido, si bien los colegios salesianos se gestionan bajo un enfoque tradicional, el Sistema Preventivo de Don Bosco les otorga una identidad única e irrepetible, teniendo siempre en cuenta que el verdadero quehacer de la escuela es para el desarrollo de los estudiantes, por ello una escuela democrática se conjuga con el funcionamiento de cada una de las Comunidades Educativas Pastorales (CEP). El Sistema Preventivo de Don Bosco, es motivo de análisis constante no sólo en cada una de las CEP si no que por su marcada trascendencia en el sentido pedagógico hace que se estudie fuera del contexto confesional. Es por ello, que más allá de lo que se expresa en la espiritualidad de Don Bosco, para efectos de la presente investigación, el sentido es vincular los elementos centrales del sistema preventivo: razón, amor y religión, con principios del liderazgo distribuido centrado en la participación de los estudiantes en el aula. Para ello es fundamental destacar lo expresado por Peraza (2009), respecto a que es necesario escoger una forma de estudiar a Don Bosco, para lograr tener un conocimiento completo y objetivo, considerando que es una persona que se insertó en la realidad de su tiempo y que por ende está sujeta a cambios personales o culturales y sociales, determinados lógicamente por el contexto. Por lo anterior, fue fundamental para el sistema, el acompañamiento y el diálogo constante, en este sentido, Lenti (2010), afirma la gran preocupación de Don Bosco por el estado material y espiritual en un equilibrio constante de ambos elementos. Todas las personas se desarrollan dentro de un contexto determinado, el cual las construye, porque el entorno inmediato determina el modo de actuar de ellas frente a determinadas problemáticas que suscita el devenir político, social, económico y cultural. Aquí radica la trascendencia del sistema preventivo, porque Don Bosco es un ser político y crítico de lo que ocurre en su época, ahí nace su idea de compromiso con los jóvenes más vulnerables, comprendió que requerían ser educados y acompañados. Complementando lo anterior, Braido (2001), plantea que el oratorio como expresión principal del sistema preventivo es una obra pedagógica, una estrategia para resolver o intentar responder a las necesidades del contexto, develando un Don Bosco preocupado por el bienestar de los jóvenes espiritual y materialmente hablando, y de ésta forma puedan insertarse en la sociedad como buenos cristianos y honestos ciudadanos Cian (2001), sostiene que es impresionante la construcción y amplia difusión que ha tenido el sistema educativo de Don Bosco al lado de grandes teóricos de pedagogía que específicamente abordan el proceso de enseñanza y aprendizaje. 
Si bien la metodología que sustenta este sistema se basa en elementos de la tradición cristiana, sobrepasó esas fronteras y sus ideas han sido tomadas e incluso extrapoladas a distintos contextos no cristianos en el mundo. De acuerdo con lo expuesto la acción de educar es incompleta e ineficaz si no se basa en la relación entre el profesor y la estudiante, construida de forma espontánea, en confianza, colaborativa y sincera considerando los intereses de cada uno de los actores que componen la comunidad. Esta relación no conlleva una crisis de autoridad que pueda sufrir el profesor, de hecho, la autoridad con la libertad siempre está en enfrentamiento. Sin embargo, el profesor se debe encargar de promover la autonomía del estudiante, ser capaz de entregarle las herramientas suficientes para su desarrollo, no dictar normas y contenidos que carecen de sentido para el contexto particular de cada uno de ellos. En esta interacción entre el profesor y los estudiantes, bajo el sistema preventivo el rol y responsabilidad del primero es trascendente en el proceso de enseñanza y aprendizaje. Russo (2007), relata la paternidad de los educadores de la época, incluso ofreciendo las casas a los jóvenes desprotegidos o que pasaban por malos momentos con su entorno familiar. Don Bosco, ejercía un liderazgo claro para lograr un objetivo, que era el bienestar de los jóvenes, los cuales convivían con una precariedad espiritual y material. Bolívar (2010), plantea que hoy en día, los líderes exitosos desarrollan una visión para sus escuelas basadas en valores personales y profesionales, es decir, objetivos individuales y organizativos, articulan esta visión en cada oportunidad e influyen sobre su equipo para que esta visión sea compartida. La relevancia del liderazgo escolar se basa en que las escuelas no pueden mejorar sin él, siendo el segundo elemento más importante luego de la enseñanza en el aula. Considerando los cimientos del sistema preventivo, es fundamental la participación de los jóvenes que pertenecen a las comunidades educativas salesianas, construidas bajo este sistema educativo. En este sentido, como el contexto sociopolítico y cultural es claramente distinto, a la asistencia se le suma el acompañamiento de los jóvenes en todo ámbito, siempre teniendo en cuenta sus expresiones. A partir de lo anterior, se establece una relación entre la participación de estudiantes en el contexto salesiano y el ejercicio del liderazgo distribuido porque son múltiples líderes los que ejercen influencia para que se logren los objetivos académicos y pastorales.

\section{METODOLOGÍA}

La investigación se desarrolla bajo el paradigma cualitativo, porque el objeto de estudio que es el aula se comprende a través de la interpretación de procesos naturales que se desarrollan en él. En este sentido, la actitud de los docentes y estudiantes se produce en un espacio que es cambiante y dinámico. Desde el paradigma cualitativo, la intención es comprender la relación entre la participación de los estudiantes y principios del liderazgo distribuido en el aula de un colegio salesiano. Desde la perspectiva de Cáceres (2003), la utilización de este enfoque permite generar conocimiento ajustado a los objetos de estudio. Dicho de otro modo, permite mayor profundidad y precisión para la interpretación de procesos que se desarrollan en su ambiente natural. Para fundamentar lo anterior, Pérez (1990) afirma que para este paradigma la realidad es global, holística y polifacética, nunca estática, por lo que no existe una realidad, sino múltiples que están interrelacionadas. Debido a ello que Olabuenaga (2003), defendiendo el paradigma cualitativo para la investigación, utiliza un proceso interpretativo para comprender la realidad, afirmando que el investigador, bajo 
este enfoque interpreta todos los sucesos a la luz de experiencias anteriores o de cualquier elemento que pueda ayudar a comprender mejor la realidad estudiada, lo que le entrega profundidad al estudio que realiza. Respecto al diseño de la investigación, se ha decidido utilizar el estudio de caso, que si bien no propone generalizar resultados cómo sí lo hacen los diseños cuantitativos, permite realizar un estudio en profundidad del fenómeno, lo que produce antecedentes prototípicos de un problema determinado para el desarrollo de futuras investigaciones. En la presente investigación, el estudio de caso se utiliza cómo un método descriptivo intensivo y global de un caso único, con el objetivo de comprender un fenómeno particular. Fundamentado esto:

El cometido real del estudio de casos es la particularización, no la generalización. Se toma un caso particular y se llega a conocerlo bien, y no principalmente para ver en qué se diferencia de los otros, sino para ver que es, qué hace (Stake, 1999, p. 20).

En cuanto a la tipología del estudio de caso, Yin (1994), desde el punto de vista del número de casos objeto de análisis, propone los tipos simples y múltiples. Por otro lado, desde el punto de vista del objetivo de la estrategia de investigación, el autor presenta los casos descriptivos y exploratorios. Considerando lo presentado, se aplica un estudio de caso único, descriptivo y exploratorio. Es único, porque se toma el caso de las interacciones y posibles participaciones de estudiantes de enseñanza media dentro del aula de un colegio salesiano y su relación con los aspectos de un liderazgo distribuido centrado en el aula. Es descriptivo, porque el objetivo es describir cómo ocurren los fenómenos dentro del aula en su contexto real. Finalmente, es exploratorio, porque si bien existe un marco teórico, en relación con el planteamiento del problema y el grado de influencia que tiene el investigador sobre el comportamiento de factores o variables (no interviene) no posee una estructura clara, es por ello que el fin es acercar la teoría con el objeto de estudio. Teniendo en consideración el enfoque y diseño, se realiza una muestra teórica que según Martínez (2006), en el estudio de caso no se selecciona una muestra representativa de una población sino una muestra teórica. Para la selección de la muestra, el objetivo era saturar el espacio simbólico del problema de investigación (aula). En consecuencia, se seleccionó una muestra teórica conformada en una parte por cuatro profesores de distintas asignaturas que ejercen su práctica educativa entre los niveles de $\mathrm{I}^{\circ}$ a $\mathrm{IV}^{\circ}$ medio y que tienen cinco años o más de experiencia desempeñándose en la organización educativa. La otra parte de la muestra está compuesta por 12 estudiantes. En este caso el objetivo es otorgar heterogeneidad en la elección debido a que se escogió a un representante por cursos que pertenece a la directiva desde primero a cuarto año de educación media. Vale decir, que la selección se realizó a partir del interés y participación voluntaria en la investigación. Lo anterior, responde a que es necesario distintos puntos de vista dentro de una lógica homogénea, considerando que todos son estudiantes de la misma institución.

Los criterios de selección para profesores fueron: de asignatura de enseñanza media, desempeño en el cargo de a lo menos cinco años y no participar con los estudiantes en ninguna actividad pastoral o fuera del ámbito formal de enseñanza. Los criterios para selección de estudiantes fueron: Pertenecer a la directiva del curso (considerando los requisitos internos para ser parte) y ser parte del cetro de estudiantes electo.

Por otra parte, para el procedimiento de recolección de datos, las técnicas que se aplicaron en este estudio fueron: entrevista estructurada en profundidad, aplicada a 
profesores y focus group aplicado a estudiantes, además de un análisis de documento. La razón del uso de estas técnicas de investigación se debió a que ellas se complementan mutuamente. Vale destacar, respecto al focus group, se realizaron dos grupos, formados cada uno por seis estudiantes, el moderador tiene una participación activa en ambos grupos, reestructurando las interrogantes a medida que avanza la conversación. Los estudiantes se distribuyeron heterogéneamente por nivel y curso, incluyendo por cada grupo dos representantes del centro de alumnos. Sin embargo, para que se cumpliera con la diversidad de opiniones, se procuró la participación de a lo menos un estudiante por nivel, de primero a cuarto año de enseñanza media. En el grupo de discusión o debate se expresan e intercambian opiniones en un contexto cotidiano, sin ser aislada la muestra del contexto y, además, permite correcciones de opiniones por parte del grupo que "no son adecuadas, no se comparten socialmente o son extremas, como medio para validar afirmaciones y visiones" (Flick, 2007 p. 129).

\section{RESULTADOS}

A partir de la triangulación de las técnicas, Primero, respecto al entendimiento del liderazgo distribuido en el aula (ENTLDA). Con el fin de aproximarse al concepto de liderazgo distribuido, fue necesario guiar la entrevista (por eso su carácter de semiestructurada), infiriendo que puede constituir una definición de mayor especificidad para los profesores. Es por ello, que entregan definiciones del concepto de liderazgo relacionado a las distintas metodologías que aplican para impartir sus asignaturas. Tomando como análisis los temas expuestos por los estudiantes en el focus group, se destacan algunos elementos fundamentales para comprender sus perspectivas. Segundo, en cuanto a la interacción entre profesores y estudiantes de un colegio salesiano (INTAULASDB). Es fundamental, primero, exponer lo expresado en el PEPS local, el cual de forma general define la CEP y sus objetivos transversales, se apega al sistema pedagógico de Don Bosco, el cual tiene como característica esencial la presencia de los educadores en medio de los estudiantes. A partir de esto, en el discurso de los profesores queda de manifiesto el valor que le otorgan al hecho de construir un ambiente propicio para que se produzcan los aprendizajes, por lo que en la cotidianeidad del proceso es un elemento primario, incluso antes de abordar temas netamente académicos. Los estudiantes, por su parte, no mencionan el factor ambiente propiamente tal como un factor determinante en la enseñanza, sino que se refieren específicamente a las relaciones que se dan entre pares. En ese sentido, comparten la idea que en general existe unión en los cursos, pero que es inevitable que en el espacio de aula se formen grupos con intereses comunes. Tercero, en cuanto a las modalidades de participación de estudiantes en el aula (PARTESTAULA). Los profesores plantean que en temas netamente académicos como salidas pedagógicas y procesos evaluativos los estudiantes no tienen incidencia en aquellas decisiones, debido a que eso está planificado preliminarmente. En temas paralelos a lo académico, que guardan relación con las normas de convivencia dentro del aula, se presentan dos miradas muy marcadas respecto a si se deben dialogar. Hay profesores que conversan con los estudiantes las normas desde el inicio del año escolar e incluso son capaces de adaptarlas a los diferentes contextos. Existen otros profesores, que, si bien son flexibles con algunas normas dentro de la sala de clases que responden a la cotidianeidad surgida en el trato con los estudiantes, no acuerdan ni 
dialogan normas referidas al reglamento interno del colegio, por lo que lo aplican sin mayor dialogo. Para los estudiantes ocurre algo muy parecido, a nivel macro, de organización, basada en el reglamento interno del establecimiento los estudiantes consideran que no tienen ninguna incidencia ni participación en aquellas normas. Sin embargo, acotándose al espacio del aula, algunos reconocen que sí se dan esas oportunidades de dialogar con el profesor ciertas normas. En cuanto al documento (PEPS) vale destacar que específicamente la participación de los estudiantes en el aula no se visualiza en la redacción del PEPS, por lo que la descripción se construye de forma implícita:

[...] Siguiendo con su fase contextual y diagnóstica situacional, a partir de cuestionarios cerrados aplicados a Alumnos, Docentes y Apoderados. A la luz de la información (Diagnóstica), se construyeron los objetivos estratégicos por el Consejo de Dirección, y se validaron a través de un cronograma de trabajo con los Consejos de Coordinación del Ciclo I y II, Consejos de Profesores, Consejo Escolar, Consejo de Presidentes de los Estudiantes y el Centro de Padres y Apoderados" (PEPS, 2015, pp. 4-5).

En el PEPS se señala como centro del proceso la figura del estudiante en un ambiente que debe ser construido para su desarrollo tanto en el ámbito académico como valórico.

Somos una Comunidad Educativo - Pastoral Salesiana por lo cual, ponemos en el centro de nuestra preocupación y trabajo a la persona del estudiante [...] En un ambiente de familia que educa, que acoge, que evangeliza de forma que los niños y jóvenes encuentran en esta CEPS su propia casa” (PEPS, 2015, p. 6).

A nivel de gestión, se manifiesta el apego a un modelo participativo para construir un proyecto común con la participación desde sus funciones de los estudiantes, padres y educadores.

Creemos en el valor de la comunidad como experiencia humana y de Iglesia, por consiguiente, nos identificamos con un modelo de gestión participativa y corresponsable; con un espíritu de familia salesiana manifestado en la acogida afectuosa que busca integrar a cada uno de nuestros estudiantes, padres y educadores en un proyecto común, en la promoción de la vida comunitaria, fraterna y solidaria y en el protagonismo de niños y jóvenes" (PEPS, 2015, pp. 6-7).

\section{DISCUSIÓN Y CONCLUSIONES}

A partir del sustento teórico sobre el liderazgo distribuido en el aula, los datos que entregan los profesores es que convergen en la idea de su transversalidad y el trabajo colaborativo, más allá de la imposición de contenidos y dictar instrucciones. En este sentido, los profesores reconocen la importancia de la distribución del liderazgo. Los estudiantes, por su parte, también otorgan importancia al liderazgo para el desarrollo de sus aprendizajes, planteando que para toda actividad que se desarrolla dentro de la organización se requiere de él, es decir, siempre visualizan la figura de un líder que debe organizar, guiar e incluso mandar los datos entregados por profesores y estudiantes es sustentada por el PEPS local, el 
cual aboga por el trabajo en equipo, basado en objetivos comunes, tiempos y modalidades de comunicación. Se apunta, en este sentido a que todos los miembros de la CEP aúnen los esfuerzos para cumplir con aquellos, desde sus propios roles y funciones dentro de la comunidad. Para ello, el documento invita a los profesores a que guíen a los jóvenes y fomenten su participación constante en temas académicos y formativos, para ello instaura el concepto de corresponsabilidad y subsidiariedad.

Respecto a la interacción entre profesores y estudiantes en el aula de enseñanza media de un colegio salesiano, desde la teoría, se comprende la acción de educar como un proceso social, lo que hace de las relaciones que se constituyen en ella algo fundamental, estimando que tanto estudiantes como profesores no se eligen unos a otros. Con este fundamento teórico, la percepción de los profesores es que valoran la interacción que se produce dentro del aula con los estudiantes, por eso se esfuerzan en generar un ambiente positivo de clase, lo que para ellos más allá de lo netamente académico es de suma importancia. Para lograr lo anterior, reconocen que fomentan el diálogo constante. Por su parte, los estudiantes, comparten la idea de que la relación es óptima y se puede dialogar constantemente sobre temas que van surgiendo día a día. El PEPS, hace carne lo referido por profesores y estudiantes, porque uno de los objetivos del sistema preventivo de Don Bosco, es la relación educativa cercana entre y la asistencia hacia los jóvenes. En este ambiente, el profesor pasa a ser un "educador pastor" que está entre.

Considerando las modalidades de participación de los estudiantes en el aula, desde la teoría se esgrime que el sistema preventivo nace por el amor del santo a los jóvenes más necesitados de la época, adquiriendo voluntariamente un compromiso por su bienestar material y espiritual. Para que se produzca lo anterior, el rol del profesor es trascendental porque se debe encargar de promover la autonomía del estudiante, ser capaz de entregarle las herramientas suficientes para su desarrollo, no dictar normas y contenidos que carecen de sentido para el contexto particular de cada uno de ellos. Los profesores reconocen que propician en el desarrollo de sus clases instancias para que los estudiantes participen y de esa forma construyan sus propios aprendizajes a través de un trabajo autónomo bajo su guía, a través de preguntas que permitan analizar desde sus propias perspectivas, comparaciones de procesos, definiciones guiadas, combinación de teoría y práctica, actividades grupales, ronda de preguntas, etc. sin embargo, aquello depende del grado de complejidad de la asignatura, tomando en cuenta que algunas requieren de mayor conocimiento de aspectos teóricos por sobre lo práctico, por lo que los conceptos previos generalmente no existen. Paralelo a lo académico, en lo relativo a generar un ambiente propicio para el aprendizaje a través de normas de convivencia, los profesores entregan dos visiones muy marcadas respecto a dialogar con los estudiantes aquellas normas. Por consiguiente, hay profesores que, si bien, ejecutan al pie de la letra el reglamento interno del colegio, dentro del aula conversan y acuerdan con los estudiantes normas básicas de convivencia, por lo general a principio del año escolar, adaptando las mismas a la realidad de cada grupo curso, lo que denota interés por la participación del estudiante. Otros profesores aplican las normas establecidas en el reglamento interno del colegio, sin generar mayor diálogo con los estudiantes respecto a su importancia en relación a las características del grupo curso, argumentando que el documento fue sociabilizado con los padres al momento de la matrícula y por ende hay un acuerdo en cumplir lo expuesto. Los estudiantes, a diferencia de los profesores, tienen una visión mucho más crítica respecto a su participación en el aula y la percepción de los docentes respecto a ella. Hacen la diferenciación entre los profesores, porque explican que 
algunos se apegan al PEPS local en cuanto a propiciar instancias de participación para los estudiantes, mientras que otros no lo realizan. Este análisis, llama la atención porque los estudiantes conocen la misión y visión del colegio a través de las principales orientaciones del documento, por lo que su percepción crítica es argumentada. Defienden la idea que la organización, al ser confesional promueve la interacción de sus miembros, siempre bajo el objetivo de la formación integral de los jóvenes, en este contexto, consideran que hay profesores que sólo se esmeran en transmitir contenidos sin considerar la formación valórica y formativa, mientras que hay otros profesores que conocen el proyecto salesiano y se relacionan de forma más cercana con los estudiantes, intentando generar un buen ambiente basado en el diálogo interactivo a través de la participación.

La principal limitación del estudio radica en que, desde la perspectiva de la escuela tradicional, cuando se reflexiona, cuestiona y discute sobre la participación de los estudiantes en el aula, ésta queda relegada a las decisiones tomadas por el profesor, el cual pasa a ser el factor excluyente para el logro de los aprendizajes. En este contexto es necesaria la interacción que se produce de forma espontánea entre él y los estudiantes, porque ayudan al primero a reflexionar sobre sus prácticas, haciéndolas más democráticas y dialógicas. En este ambiente de participación, el liderazgo distribuido cumple un rol fundamental, porque si bien es un factor significativo para la dirección de procesos académicos y administrativos, en el presente estudio su importancia radica en su expresión dentro del aula, entendiendo que por un lado invita a los estudiantes y profesores a trabajar para lograr los aprendizajes y, por otro lado, mejora las relaciones interpersonales dentro del espacio en el cual conviven a diario. En el contexto del sistema pedagógico salesiano, cuando el objetivo es la educación integral de los estudiantes, considerando el desarrollo valórico como cognitivo, la participación de los estudiantes es primordial, por lo que el ejercicio del liderazgo distribuido armoniza con las características del proyecto educativo propuesto por la congregación, el cual busca hacer partícipe responsablemente a todos los miembros de las comunidades educativas pastorales. En este sentido, "Hablar de participación autentica es preguntar quién participa, en qué áreas bajo qué condiciones y también participación ¿para qué; con qué fin?” (Anderson, 2001, p. 8). A partir de esto, se concluye exploratoriamente en relación al problema de investigación que como esencia del liderazgo distribuido dentro del aula se sitúa la participación de los estudiantes a partir de la interacción constante dentro del aula, lo que favorece la construcción de sus propios aprendizajes, lo que armoniza con los principales lineamientos que constituyen el PEPS local. Con esto se rompe la perspectiva tradicional de la escuela donde la participación de los estudiantes queda relegada a decisiones que emanan de la figura del profesor sin mayor diálogo ni participación de los estudiantes. Lo expresado se fundamenta en primer lugar en la manifestación de principios del liderazgo distribuido en el aula, desde la perspectiva de profesores y estudiantes y lo escrito en el PEPS. En este sentido, tanto profesores como estudiantes entregan definiciones sobre el concepto de liderazgo distribuido que se relacionan con sus prácticas dentro del aula. Los profesores relacionan el concepto con sus metodologías de clase y le otorgan importancia porque invita al trabajo colaborativo y la transversalidad de las relaciones dentro del aula, cada uno cumpliendo sus funciones para el logro de los aprendizajes. Los estudiantes, también le entrega un valor, enfatizando que todos pueden formarse como líderes en la escuela y plantean la necesidad de que surjan nuevos líderes, que sepan guiar al resto y ayudar a todos al logro de los aprendizajes, debido a sus relaciones dentro del aula y las realidades de cada grupo curso, los estudiantes convergen 
en la idea que el liderazgo distribuido permite el desarrollo de las capacidades individuales para objetivos grupales. Lo expuesto por profesores y estudiantes se refleja en el PEPS, bajo el concepto de corresponsabilidad, donde cada miembro de la CEP, bajo su función se responsabiliza en la tarea de lograr aprendizajes académicos y el desarrollo espiritual de los jóvenes, entendiendo que el objetivo es la formación integral de ellos en lo académicocognitivo y lo valórico-espiritual. En el PEPS, se identifican elementos o principios que promueven la participación de los estudiantes a través de los órganos colegiados como el centro de estudiantes, pero también en el protagonismo que deben tener para sus propios aprendizajes, dialogando con los profesores temas recurrentes que surgen en la vida en las aulas. En este sentido, destacar que el documento no es explícito en cuanto a la forma de hacerlo, es más bien una invitación a ser agentes activos en las CEP. Debido a ello, se afirma que existe una armonía implícita respecto a los principios del liderazgo distribuido en el aula de enseñanza media y lo que expresa el PEPS respecto a la participación.

\section{REFERENCIAS BIBLIOGRÁFICAS}

Ahumada, L., González, Á., Pino, M. y Maureira, O. (2017). Liderazgo distribuido en establecimientos educacionales: Recurso clave para el mejoramiento escolar. Lidereseducativos.cl. Recuperado de https://www.lidereseducativos.cl/wp content/uploads/2017/12/IT7_LA_L2_12-12.pdf

Anderson, G. (2001). Hacia una participación auténtica: deconstruyendo los discursos de las reformas participativas en educación. ( $1^{\circ}$ ed.). Nuevas tendencias en políticas educativas.

https://sites.google.com/site/unaeducacionigualitaria/home/reflexiones-\%20sobre-lecturas/haciauna-participacion-autentica-deconstruyendo-los-\%20discursos-de-las-reformas-participativasen-educacion

Aravena, F. Escare, K. y Ramírez, J. (2019). La participación democrática y la voz de los estudiantes: ¿Qué pueden hacer los líderes escolares? (Nota Técnica $\mathrm{N}^{\circ} 2$. Centro de Liderazgo para la Mejora Escolar). https://www.lidereseducativos.cl/recursos/la-participacion- democratica-y-lavoz-de-los-estudiantes-que-pueden-hacer-los-lideres- escolares/

Bertoglia, L. (2005). La interacción profesor-alumno. Una visión desde los procesos atribucionales. ( $4^{\circ}$ ed.). Escuela de Psicología Pontificia Universidad Católica de Valparaíso. https://www. psicoperspectivas.cl/index.php/psicoperspectivas/article/v iewFile/26/26

Bolívar, A. (2010). El liderazgo educativo y su papel en la mejora: una revisión actual de sus posibilidades y limitaciones. ( $2^{\circ}$ ed.). Psicoperspectivas. https://scielo.conicyt.cl/pdf/psicop/v9n2/art02.pdf

Braido, P. (2001). Prevenir, no reprimir; el sistema preventivo de Don Bosco. CCS.

Bretones, A. (1996). Concepciones y prácticas de participación en el aula según los estudiantes de magisterio. Departamento de Didáctica y Organización Escolar Universidad Complutense. https:// www.researchgate.net/publication/39156540_Concepciones_y_practicas_de_participacion_en_ el_aula_segun_los_estudiantes_de_magisterio

Cáceres, P. (2003). "Análisis Cualitativo del contenido: una alternativa metodológica alcanzable". Revista Escuela de Psicología, Vol. II, pp. 53-82. Pontificia Universidad Católica, Santiago, Chile.

Cayulef, C. (2007). El Liderazgo Distribuido una apuesta de dirección escolar de calidad. $5^{\circ}$ ed. Madrid, España: REICE. Recuperado de http://www.redalyc.org/pdf/551/55121025021.pdf

Cian, L. (2001). El sistema educativo de Don Bosco. ( $3^{\circ}$ ed.). CCS

Darling-Hammond, L. (2001). El derecho de aprender. ( $1^{\circ} \mathrm{ed}$.). Ariel.

Duverger, M. (1972). Métodos de las ciencias sociales. Ariel.

Escobar, M. (2015). Influencia de la interacción alumno-docente en el proceso enseñanza. ( $5^{\circ}$ ed.). Revista de Tecnología y Sociedad. http://www.udgvirtual.udg.mx/paakat/index.php/paakat/ article/view/23 0/347 
Estudios Pedagógicos XLVI N 3: 167-180, 2020

ESTUDIO DE CASO ÚNICO SOBRE LA PARTICIPACIÓN DE ESTUDIANTES EN EL AULA DESDE LOS PRINCIPIOS DEL LIDERAZGO DISTRIBUIDO A TRAVÉS DE FOCUS GROUPY ENTREVISTA SEMIESTRUCTURADA APLICADA...

Elmore, R. (2010). Mejorando la escuela desde la sala de clases. ( $1^{\circ}$ ed.). Área de Educación Fundación Chile. https://fch.cl/wp- content/uploads/2012/08/Libro_Elmore.pdf

Flick, U. (2007). Introducción a la investigación cualitativa. Ediciones Morata.

Jurado, C. (2009). La participación educativa del alumnado. (23ํำ ed.). Revista innovación y experiencias educativas. https://archivos.csif.es/archivos/andalucia/ensenanza/revistas/csicsif/ revista/pdf/Numero_23/CARMEN_JURADO_GOMEZ02.pdf

Kerlinger, F. (1999). Investigación del comportamiento. McGraw-Hill.

Lechner, N. (2004). Cultura juvenil y desarrollo humano. ( $8^{\circ}$ ed.). Ventana Central: Sociedad de la información y nuevas identidades. https://educiac.org.mx/pdf/Biblioteca/Juventud_e_ Identidad/002Cultura_juvenil_DH_Norbert_Lechner.pdf

Leithwood, K. (2009). ¿Cómo liderar nuestras escuelas? Aportes desde la investigación. (1ed.). Área de Educación Fundación Chile. http://ww2.educarchile.cl/UserFiles/P0038/File/libros/ Libro_Liethwood.pdf

Lenti, A. (2010). Don Bosco, historia y carisma. CCS.

Martínez, P. (2006). El método de estudio de caso: estrategia metodológica de la investigación científica. (20 ed.). Pensamiento \& Gestión. https://www.redalyc.org/pdf/646/64602005.pdf

Maturana, H. (2001). Emociones y lenguaje en educación y política. (10 ed.). Dolmen Ensayo. http:// turismotactico.org/proyecto_pologaraia/wp-content/uploads/2008/01/emociones.pdf

Maureira, Ó. (2017). Prácticas del liderazgo educativo: Una mirada evolutiva e ilustrativa a partir de sus principales marcos, dimensiones e indicadores más representativos. $1^{\circ}$ ed. Costa Rica: Revista Educación. Recuperado de: https://www.redalyc.org/jatsRepo/440/44051918001/html/index.html\#fn

Maureira, Ó., Garay, S. y López, P. (2015). Reconfigurando el sentido del liderazgo en organizaciones escolares contemporáneas: La perspectiva del liderazgo distribuido. (vol. 27.). Revista Complutense de Educación https://revistas.ucm.es/index.php/RCED/article/viewFile/47079/48402

Murillo, J. y Martínez, C. (2018). Factores de aula asociados al desarrollo integral de los estudiantes: Un estudio observacional. ( $1^{\circ} \mathrm{ed}$.). Estudios Pedagógicos.

https://scielo.conicyt.cl/pdf/estped/v44n1/0718-0705-estped-44-01- 00181.pdf

Olabuenaga, J. (2003). Metodología de la investigación cualitativa. ( $3^{\circ}$ ed.). Universidad de Deusto. http:// www.webquestcreator2.com/majwq/public/files/files_user/41661/05.\%20Metodolog\%C3\%ADa\%20 de\%20la\%20Investigaci\%C3\%B3n\%20Cualitativa.pdf

Peraza, F. (2009). Perfil sacerdotal de Don Bosco. CCS

Pérez, G. (1990). Investigación Cualitativa. Retos e interrogantes. (I-II). La Muralla.

Prieto, M. (2005). La participación de los estudiantes: ¿un camino hacia su emancipación? http://www. ubiobio.cl/theoria/v/v14/a3.pdf

Robinson, V., Lloyd, C. \& Rowe, K. (2012). El impacto del liderazgo en los resultados de los estudiantes: Un análisis de los efectos diferenciales de los tipos de liderazgo. ( $4^{\circ}$ ed.). REICE. https://revistas.uam. es/index.php/reice/article/viewFile/2835/3052

Rodríguez, G. (1996). Metodología de la investigación cualitativa. Ediciones Aljibe.

Russo, C. (2007). Don Bosco encuentra a los jóvenes. el secreto del sistema educativo de Don Bosco. (2 ed.). CCS.

Spillane, J. y Ortiz, M. (2016). Perspectiva distribuida del liderazgo y la gestión escolar: implicancias cruciales. $\left(2^{\circ}\right.$ ed). José Weinstein. http://cedle.cl/wp-content/uploads/2018/03/Liderazgo- Educativoen-la-Escuela.-Nueve-miradas.pdf

Seashore, K. (2017). Implicancias del liderazgo escolar en la efectividad de las escuelas. ( $1^{\circ}$ ed.). Ediciones Universidad Diego Portales. http://cedle.cl/wp-content/uploads/2019/03/Mejoramiento-y-Liderazgoen-la-escuela.- Once-miradas.pdf

Stake, R. (1999). Investigación con Estudios de casos. Morata.

Weinstein, J. (2019). La Confianza: Factor Clave Para Construir La Buena Escuela. Recuperado de: https:// ubiqq.com/cedle/confianza-factor-clave-para-construir-buena-escuela

Yin, R. (1994). Investigación sobre estudio de casos. Diseño y métodos. (2ºd.). SAGE. 